Published in:Ferroelectrics, Volume326,

October 2005 , pages $103-108$ http://dspace.nitrkl.ac.in/dspace

$R$ Mazumder is presently working with National Institute of Technology Rourkela

ranabrata@nitrkl.ac.in

\title{
Effect of Boron Addition on the Dielectric Properties of Giant Dielectric $\mathrm{CaCu}_{3} \mathrm{Ti}_{4} \mathrm{O}_{12}$
}

\author{
RANABRATA MAZUMDER, ANSHUMAN SEAL, \\ AMARNATH SEN, ${ }^{*}$ AND HIMADRI SEKHAR MAITI
}

Electroceramics Division, Central Glass \& Ceramic Research Institute, Kolkata 700032

\begin{abstract}
The recently discovered giant dielectric $\mathrm{CaCu}_{3} \mathrm{Ti}_{4} \mathrm{O}_{12}$ (CCTO) has been reported to show dielectric constant value as high as 80,000 for single crystals and around 10,000 for ceramics. However the dielectric constant is also associated with high dissipation factor. In the present study, it has been observed that the loss factor of CCTO can be reduced by $\mathrm{B}_{2} \mathrm{O}_{3}$ addition. The low frequency dispersion of CCTO ceramics, which indicates Maxwell-Wagner type relaxation, is reduced by boron addition. Also the temperature dependence of dielectric constant is minimized by boron addition. From the present work it can be surmised that $\mathrm{B}_{2} \mathrm{O}_{3}$ addition can favourably modify the dielectric properties of CCTO ceramic for its practical applications as a capacitor material.
\end{abstract}

Keywords $\mathrm{CaCu}_{3} \mathrm{Ti}_{4} \mathrm{O}_{12}$; dielectric; ferroelectric

\section{Introduction}

Recently, $\mathrm{CaCu}_{3} \mathrm{Ti}_{4} \mathrm{O}_{12}$ (CCTO) has generated a lot of interest because of its giant dielectric constant [1-4]. Static dielectric constant $(\varepsilon)$ value reaching around 80,000 for single crystals has been reported [1-2]. Such high $\varepsilon$ is nearly constant over a wide temperature range 100-600 K. However, the dynamic $\varepsilon$ falls from its huge static value to a low value with increasing frequency [2]. Similarly, below $100 \mathrm{~K}, \varepsilon$ value drops rapidly [1]. The possible origin of such giant dielectric constant is still controversial. CCTO has an unusual perovskite type crystal structure in which the $\mathrm{TiO}_{6}$ octahedra are strongly tilted, giving rise to small, square planar coordinate sites [5] for $\mathrm{Cu}$. Incidentally, no phase transition or peak dielectric constant characteristics of ferroelectric behaviour [6] has been found for CCTO. Also no diffused peak or nanodomain has been observed ruling out relaxor like behaviour. Indeed, $\mathrm{Ca} / \mathrm{Cu}$ sublattice is well ordered because the diffraction failed to show any broadening due to superstructure or reduced Bragg intensities. Initially, it was suggested [1-2] that the observed high $\varepsilon$ is an instrinsic effect and arises out of local dipole moments associated with the off-centre displacement of Ti ions but the transition to a ferroelectric state is frustrated by the $\mathrm{TiO}_{6}$ octrahedral tilt required to accommodate the square planar arrangement of $\mathrm{Cu}^{2+}$. However, according to the theoretical calculations of $\mathrm{He}$ et al. [7] it is difficult to provide any intrinsic mechanism of giant dielectric response of CCTO. Subramanian et al.

Paper originally presented at AMF-4, Bangalore, India, December 12-15, 2003; received in final form April 10, 2005.

*Corresponding author. E-mail: asen@cgcri.res.in 
[5] suggested that barrier layer mechanism may give rise to such a high $\varepsilon$ value of CCTO, especially considering the fact that CCTO single crystals are heavily twinned due to lack of fourfold symmetry. Impedance spectroscopy [8] of CCTO suggests the role of internal barrier layer capacitance in generating such a huge $\varepsilon$ value. In such extrinsic mechanism of giant $\varepsilon$, it is assumed that the CCTO gains are semiconducting and are divided by insulating grain boundaries. However, there still lie difficulties in explaining the rapid suppression of $\varepsilon$ at low temperature. Recently, Ramirez et al. [9] proposed an intrinsic mechanism of giant $\varepsilon$ arising out of relaxing $\mathrm{TiO}_{6}$ octahedra due to de-bracing of rigid $\mathrm{CaCu}_{3} \mathrm{Ti}_{4} \mathrm{O}_{12}$ structure because of the presence of a small concentration of defects. However, Cohen et al. [10] still argues in favour of extrinsic effect arising out of either twin boundaries, antiphase boundaries or some sort of deviation in calcium ordering.

Such high $\varepsilon$ materials are very promising for miniaturization in microelectronics and microwave devices. This is specifically true given the fact that standard barrier layer capacitors need complex fabrication procedure[11], whereas CCTO can be fabricated by a single step heat treatment in air. However, the dielectric properties of CCTO are very much dependent on the fabrication parametres $[8,12]$. Depending on the preparation conditions, some workers $[13,14]$ have even reported much lower $\varepsilon$ values of their CCTO samples. Incidentally, reported high $\varepsilon$ values are also associated with high dissipation factor (DF). For practical applications of CCTO, along with high $\varepsilon$, low DF is also of paramount importance. In the present work, an attempt has been made to lower the DF of CCTO ceramics by $\mathrm{B}_{2} \mathrm{O}_{3}$ addition (as boric acid) without compromising much the dielectric constant of the ceramics.

\section{Experimental}

To synthesize $\mathrm{CaCu}_{3} \mathrm{Ti}_{4} \mathrm{O}_{12}$, stoichiometric amounts of reagent grade $\mathrm{CaCO}_{3}, \mathrm{CuO}, \mathrm{TiO}_{2}$ were mixed thoroughly in a ball mill in ethanol medium for $24 \mathrm{~h}$. The mixture was calcined at $1000^{\circ} \mathrm{C} / 10 \mathrm{~h}$ followed by grinding for $30 \mathrm{~min}$. The powder was then characterized by XRD (Philips). Boric acid (0-10 wt \%) was mixed with the powder and pellets were made at $350 \mathrm{MPa}$ pressure and sintered at $1070^{\circ} \mathrm{C} / 20 \mathrm{~h}$. The densities of the sintered pellets were measured geometrically. The ground pellets were also characterized by XRD. The microstructures of the polished and thermally etched samples were viewed by a SEM (Leo 430i). The samples for electrical testing were polished and coated with silver paint and cured at $600^{\circ} \mathrm{C} / 10 \mathrm{~min}$. The dielectric measurements were made in a Hioki 3532-50 LCR Hitester in the frequency range of $100 \mathrm{~Hz}-1 \mathrm{MHz}$ from room temperature to $170^{\circ} \mathrm{C}$.

\section{Results and Discussion}

From the X-ray diffractograms (Fig. 1), it is observed that up to $5 \mathrm{wt} \%$ boric acid addition (equivalent to $2.8 \% \mathrm{~B}_{2} \mathrm{O}_{3}$ ), there is no significant change in the peak positions with respect to that of pure CCTO. For $7 \mathrm{wt} \%$ (equivalent to $3.9 \% \mathrm{~B}_{2} \mathrm{O}_{3}$ ) and $10 \mathrm{wt} \%$ (equivalent to $5.6 \% \mathrm{~B}_{2} \mathrm{O}_{3}$ ) boric acid addition, no additional peak is observed though there is a slight high angle shift of the diffraction peaks indicating some boron doping in CCTO. The most probable site of $\mathrm{B}^{3+}$ replacement, considering its small ionic size, is the $\mathrm{Ti}^{4+}$ site in CCTO. The charge compensation [12] due to boron substitution can be of either electronic type (where some $\mathrm{Ti}^{4+}$ is converted to $\mathrm{Ti}^{3+}$ ) or ionic type (formation of oxygen vacancies). As the dissipation factors of the samples decrease with boron addition (discussed later), the charge compensation mechanism is probably ionic type. Hence, the presence of oxygen 


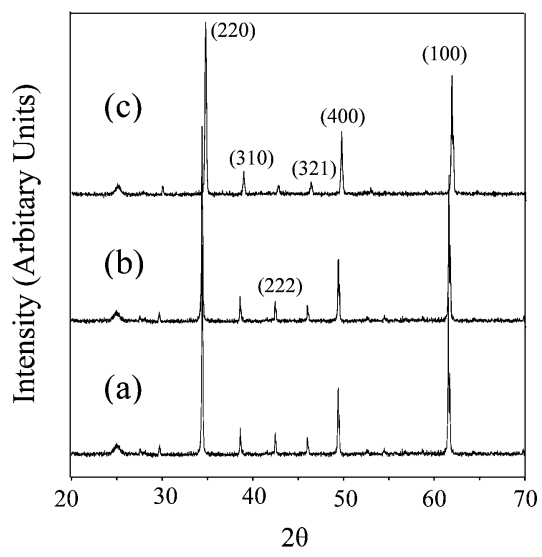

Figure 1. X-Ray diffractograms of CCTO ceramics containing (a) $0 \mathrm{wt} \%$, (b) $5 \mathrm{wt} \%$, and (c) $10 \mathrm{wt} \%$ $\mathrm{H}_{3} \mathrm{BO}_{3}$.

vacancies lower the lattice dimensions with a consequent high angle shift of the X-ray diffraction peaks.

Figure 2 depicts the effect of boric acid addition on the sintered density of the pellets. In the present investigation, the true density of CCTO powder was calculated by the standard method [15] using a specific gravity bottle and kerosene medium. The true density was found to be $4.9 \mathrm{gcm}^{-3}$. Initially, $\mathrm{B}_{2} \mathrm{O}_{3}$ addition improved the bulk density slightly. However, for 7 and $10 \mathrm{wt} . \%$ boric acid addition, the sintered density decreased. The addition of $\mathrm{B}_{2} \mathrm{O}_{3}$ is expected to help in liquid phase sintering [16]. However, for higher amounts of $\mathrm{B}_{2} \mathrm{O}_{3}$, the densification again decreased because there should be an optimum amount of liquid phase above which densification is inhibited due to the formation of a thick coating of the liquid phase around the grains [17].

The effect of $\mathrm{B}_{2} \mathrm{O}_{3}$ addition on the microstructure of the sintered pellets has been shown in Figs. 3(a)-(c). The pellets containing higher amounts of $\mathrm{B}_{2} \mathrm{O}_{3}$ showed lowered average grain size and more porosity. The formation of a thick grain boundary phase due to boric acid addition is also reflected from the SEM micrograph (Fig. 3(d)) indicating the formation of a liquid phase during sintering.

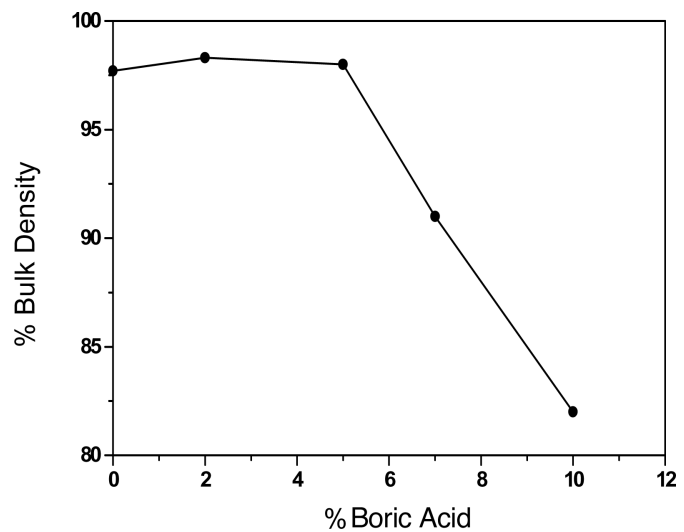

Figure 2. Effect of boric acid addition on the sintered density of CCTO ceramics. 

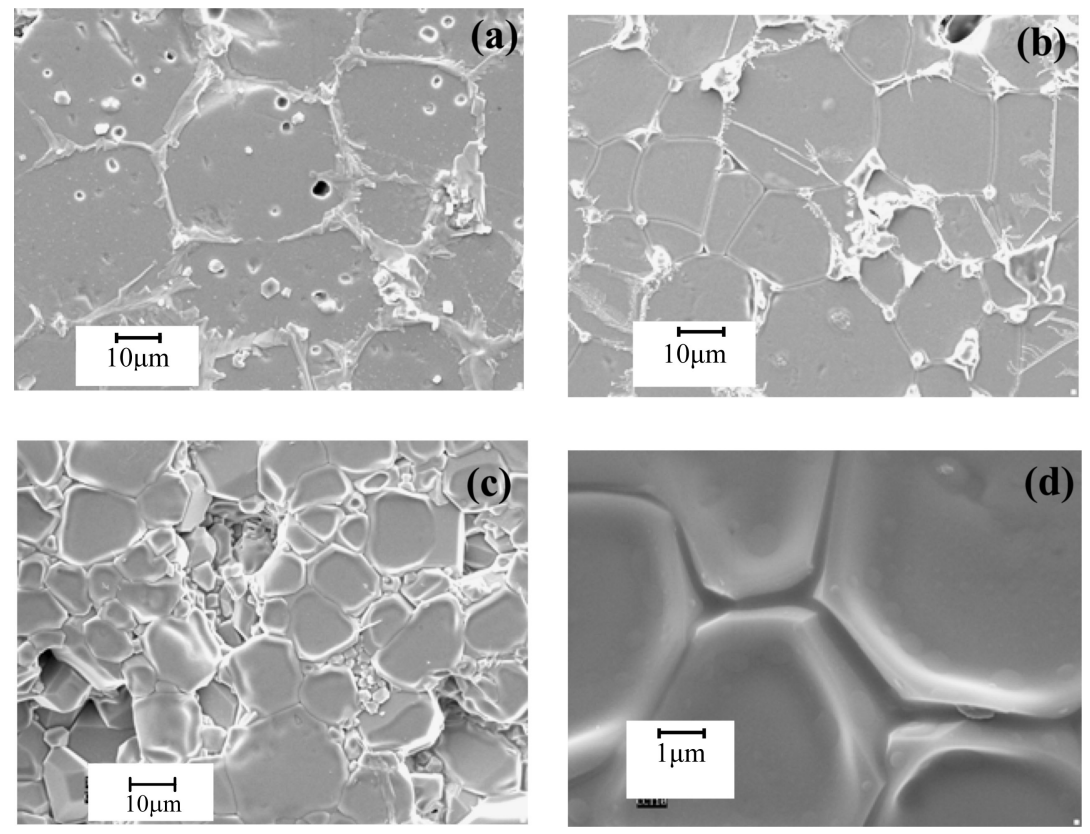

Figure 3. SEM micrographs of (a) pure CCTO ceramics (b) CCTO made with 5 wt $\%$ boric acid addition (c) CCTO made with $10 \mathrm{wt} \%$ boric acid addition and (d) CCTO made with $10 \mathrm{wt} \%$ boric acid addition where a clear grain boundary phase can be observed.

From Fig. 4(a), it is evident that boron addition drastically lowers the low frequency dielectric constant of CCTO but at high frequencies (especially at $1 \mathrm{MHz}$ ) the lowering of $\varepsilon$ is marginal. It may be noted that in the present case, at low frequencies, the $\varepsilon$ values for pure CCTO are much higher than those of similar polycrystalline samples reported by other workers $[1,2,8]$. This may be due to some variation in processing conditions which dramatically affects $[8,12]$ the dielectric properties of CCTO ceramics. Also it has been observed that the effect of $\mathrm{B}_{2} \mathrm{O}_{3}$ addition on the dielectric properties of CCTO ceramics is prominent at the low frequencies. Along with the lowering of $\varepsilon, \mathrm{B}_{2} \mathrm{O}_{3}$ addition also lowers the DF (Fig. 4(b)). However, at high frequencies (near $1 \mathrm{MHz}$ ), the DF for all the compositions shows a sharp rising trend as observed by other workers [13]. The sharp rise in DF is probably a resonance effect [19] arising from the stray inductance of the contacts and leads. The capacitance of the samples being of the order of $10 \mathrm{nF}$ and a stray inductance of the order of a few micro-Henry [20] can induce L-C resonance in the $\mathrm{MHz}$ frequency range. This is also supported by the rise of impedance phase angle (Fig. 4(c)) towards positive direction at around $1 \mathrm{MHz}$ frequency indicating the approach of resonance. It is evident from the Fig. $4(\mathrm{a} \& \mathrm{~b})$ that the $\varepsilon$ and DF values for pure CCTO and CCTO prepared by adding 2 and $5 \mathrm{wt} \%$ boric acid show low frequency dispersion. In this case, the giant dielectric constant is also associated with a high loss (DF). Incidentally, the DF tends to increase without showing a peak at low frequencies which is the signature of Maxwell-Wagner type relaxation [18].

However, 7 and 10 wt.\% boric acid additions in CCTO lower the $\varepsilon$ values and reduce the low frequency dispersion. This is probably due to a) formation of a thicker grain boundary layer (Fig. 3(d)) for samples containing higher amounts of $\mathrm{B}_{2} \mathrm{O}_{3}$ leading to poor effective capacitance arising out of brick-work layer effect from grain and grain-boundary 

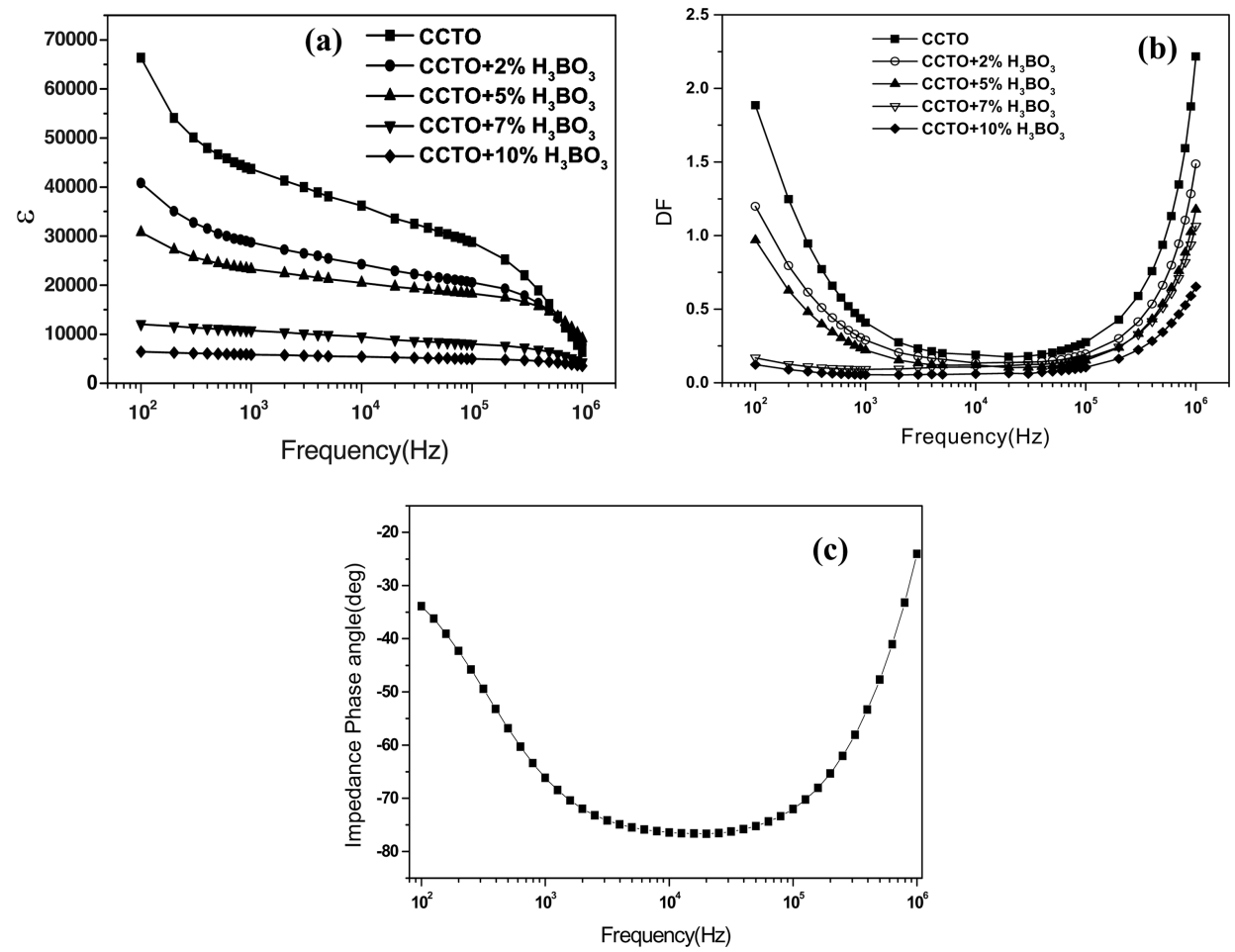

Figure 4. Variation of (a) dielectric constant (b) dissipation factor with frequency for CCTO ceramics prepared by adding different amounts of boric acid. (c) Variation of impedance phase angle with frequency for pure CCTO ceramics.

combination and b) modification(s) inside the grains structure due to boron incorporation affecting the overall capacitance of the grains. Incidentally, the rise of DF (Fig. 5(b)) with temperature (which is more for pure CCTO) indicates the thermally activated nature (increase ac conductivity with frequency) of dissipation.
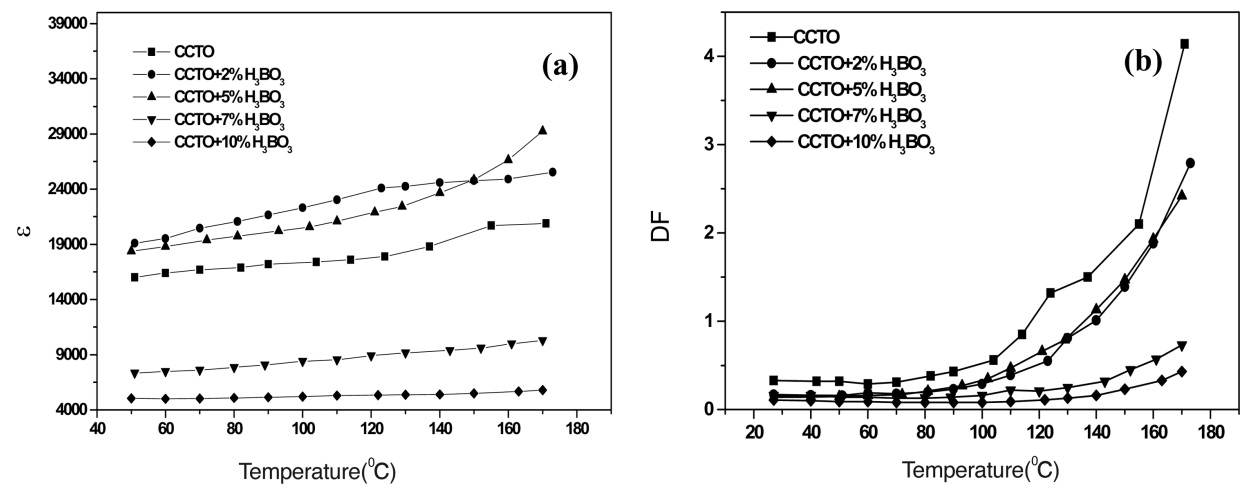

Figure 5. Temperature dependence of (a) dielectric constant (at $100 \mathrm{kHz}$ ) and (b) dissipation factor (DF) of CCTO ceramics prepared by adding different amounts of boric acid. 
Another positive aspect of $\mathrm{B}_{2} \mathrm{O}_{3}$ added CCTO ceramics is their temperature stability of dielectric properties (Figs. 5(a) \& (b)), which is one of the important requirements for practical applications of CCTO as a capacitor material.

\section{Conclusion}

It has been observed that the loss factor of giant dielectric CCTO can be reduced by $\mathrm{B}_{2} \mathrm{O}_{3}$ addition. However, boron addition lowers the dielectric constant of CCTO. At the same time, the low frequency dispersion of CCTO and the temperature dependence of dielectric constant are also reduced by boron addition. From our study, it can be surmised that CCTO prepared with $\mathrm{H}_{3} \mathrm{BO}_{3}$ addition (especially $7 \mathrm{wt} . \%$ ) can be a good candidate for capacitor applications because of its high $\varepsilon$ value (above 10,000) which is nearly independent of frequency and temperature. At the same time its DF is much lower than that of pure CCTO.

\section{Acknowledgment}

One of the authors (R.M.) is thankful to CSIR India for financial support. The authors thank all the staff members of Electroceramics Division for rendering help in carrying out this work.

\section{References}

1. A. P. Ramirez, M. A. Subramanian, M. Gardel, G. Blumberg, D. Li, T. Vogt, and S. M. Shapiro, Solid. St. Commun. 115, 217 (2000).

2. C. C. Homes, T. Vogt, S. M. Shapiro, S. Wakimoto, and A. P. Ramirez, Science 293, 673 (2001).

3. A. F. L Almeida, R. S. deOliveira, T. C. Goes, J. M. Sasaki, A. G. Souza Filho, J. Mendes Filho, and A. S. B. Sombra, Mats. Sc \& Engg. B 96, 275 (2002).

4. M. A. Subramanian and A. W. Sleight, Solid St. Sci. 4, 347 (2002).

5. M. A. Subramanian, L. Dong, N. Duan, B. A. Reisner, and A. W. Sleight, J. Solid St. Chem. 151, $323(2000)$.

6. S. M. Moussa and B. J. Kennedy, Mat. Res. Bull. 36, 2525 (2001).

7. L. He, T. B. Neaton, M. H. Cohen, D. Vanderbilt, and C. C. Homes, Phys. Rev. B 65, 214112 (2002).

8. D. C. Sinclair, T. B. Adams, F. D. Morrison, and A. R.West, Appl. Phys. Lett. 80(12), 2153 (2002).

9. A. P. Ramirez, G. Lawes, V. Butko, M. A. Subramanian, and C. M. Varma, arxiv.org/cond.-mat/ 0209498

10. M. H. Cohen, J. B. Neaton, L.He, and D. Vanderbilt, J. Appl. Phys. 94(3), 3229 (2003).

11. A. J. Moulson and J. M. Herbert, Electroceramics: Materials, Properties and Applications, Chapman and Hall, London (1990).

12. A. R. West, T. B. Adams, F. D. Morrison, and D. C. Sinclair, J. Euro.Ceram.Soc. 24, 1439 (2004).

13. P. Jha, P. Arora, and A. K. Ganguli, Mats. Lett. 57, 2443 (2003).

14. L. C. Kretly, A. F. L. Almeida, R. S. DeOlivera, J. M. Sasaki, and A. S. B. Sombra, Microwave and Optical Tech Lett 39(2), 145 (2003).

15. R. W. Grimshaw, The Chemistry and Physics of clays, London, (1971).

16. N. Halder, D. Chattopadhyay, A. Das Ssharma, D. Saha, A. Sen, and H. S. Maiti, Mat. Res. Bull. 36, 905 (2001).

17. R. M. German, Sintering Theory \& Practice, John Wiley \& Sons, New York (1996).

18. D. O'Neill, R. M. Bowman, and J. M. Gregg, Appl. Phys. Lett. 77(10), 1520 (2000).

19. P. C. Joshi and S. B. Desu, J. Appl. Phys. 80(4), 2349 (1996).

20. C. H. Lu and C. Y. Wen, Mats. Lett. 38(4), 278 (1999). 